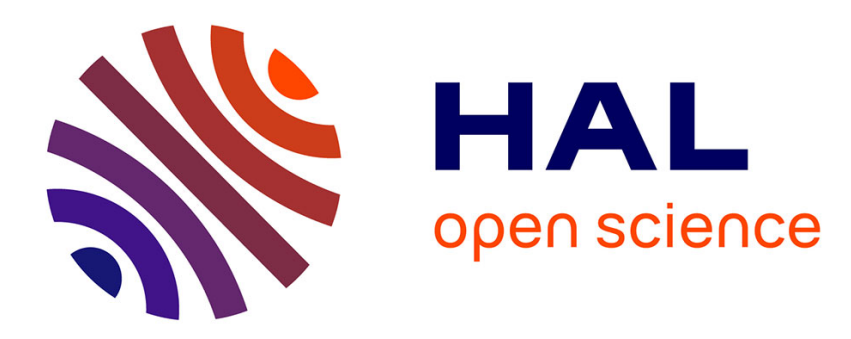

\title{
Effects of the growth mechanism on the structure of aggregation clusters
}

\author{
M. Kolb
}

\section{To cite this version:}

M. Kolb. Effects of the growth mechanism on the structure of aggregation clusters. Journal de Physique Lettres, 1985, 46 (14), pp.631-637. 10.1051/jphyslet:019850046014063100 . jpa-00232572

\section{HAL Id: jpa-00232572 https://hal.science/jpa-00232572}

Submitted on 1 Jan 1985

HAL is a multi-disciplinary open access archive for the deposit and dissemination of scientific research documents, whether they are published or not. The documents may come from teaching and research institutions in France or abroad, or from public or private research centers.
L'archive ouverte pluridisciplinaire HAL, est destinée au dépôt et à la diffusion de documents scientifiques de niveau recherche, publiés ou non, émanant des établissements d'enseignement et de recherche français ou étrangers, des laboratoires publics ou privés. 
Classification

Physics Abstracts

$05.40-82.70-68.70$

\title{
Effects of the growth mechanism on the structure of aggregation clusters
}

\author{
M. Kolb \\ Laboratoire de Physique des Solides (*), Bât. 510, Université Paris-Sud, Centre d’Orsay, \\ 91405 Orsay Cedex, France
}

(Reçu le 27 mars 1985, accepté sous forme définitive le 29 mai 1985)

\begin{abstract}
Résumé. - Les corrélations angulaires sont calculées à l'intérieur des amas obtenus par collage de particules (PA) et par collage d'amas (CA). Alors que les amas sont isotropes pour CA, ils sont anisotropes pour PA. Les exposants de la fonction de corrélation en deux dimensions, parallèle et perpendiculaire à la direction de croissance, diffèrent de $\Delta A=0,16 \pm 0,05$ pour PA. Ceci introduit des corrections aux lois d'échelle.

Abstract. - Angular correlations are calculated inside aggregation clusters of diffusion limited particle (PA) and cluster (CA) aggregation. The aggregates are isotropic for CA but anisotropic for PA, scaling differently parallel and perpendicular to the direction of growth. The correlation function exponents in two dimensions differ by $\Delta A=0.16 \pm 0.05$ in the two directions. This leads to correction to scaling effects, especially for growth on a lattice.
\end{abstract}

The fractal dimension is an important measure for the many growth processes that have been investigated in recent years [1]. It has led to a classification of the resulting aggregates in terms of their scaling properties. However, this concept - the way it was applied - could not give any information how the structure was grown. Clusters grown in quite different ways can have the same fractal exponent (as good as one can tell) though they clearly are not identical.

Here, I calculate angular correlation functions for diffusion limited particle and cluster aggregation. This is motivated by the observation, that in PA the seed particle is the centre of growth, whereas in CA there is no fixed centre in the growing clusters. Angular correlations are the simplest way to test if a cluster has a bias with respect to its centre.

The results show that CA clusters are isotropic except for slight lattice effects, but PA clusters show a clear anisotropy with respect to the centre. The correlations orthogonal to the direction of growth fall off with a slightly larger exponent than the ones parallel to it. The smallness of this exponent explains why visually the clusters appear internally isotropic on all but the largest scales.

PA [2] and CA [3] are two simple models introduced to describe diffusion limited growth processes related to dielectric breakdown, electrodeposition, colloids and aerosols [1]. In PA,

$\left(^{*}\right)$ Laboratoire associé au CNRS. 
a particle is released far from a seed particle and moves randomly through space until it sticks to the seed. Then, another particle is released, it diffuses until it sticks to the two particles, etc... In CA (hierarchical version [4]) particles diffuse pairwise until they stick together, then such small clusters diffuse pairwise until they stick, etc...

Here 100 clusters were grown in two dimensions on a square lattice, with 5000 particles for PA respectively, 4096 particles for CA. The correlations $c(\rho, r)$ are defined as follows (Fig. 1) : if $\boldsymbol{\rho}$ is the coordinate of a particle of the cluster, then $c(\boldsymbol{\rho}, \mathbf{r})=\langle n(\boldsymbol{\rho}+\mathbf{r})\rangle$ is the average density at $\mathbf{r}$, measured from the particle at $\boldsymbol{\rho}$. In the scaling region, where

$$
1 \ll r \ll \rho \ll R
$$

$c(\boldsymbol{\rho}, \mathbf{r})$ only depends on $r$ and $\theta$, the angle between $\mathbf{r}$ and the direction of growth. $R$ is the radius of the cluster. On a lattice, there is an additional dependency on $\alpha$, the angle between $\rho$ and one axis of the lattice. Two types of correlations have been calculated : axial correlations $(\theta=\alpha)$ and diagonal correlations $(\theta=\alpha-\pi / 4)$, both as a function of $r$. In both cases, $\theta=0$ corresponds to the direction radially outward from the centre. The lattice effects of the square lattice are seen best when comparing axial and diagonal correlations. Because of symmetry, the angles can be restricted to $0 \leqslant \theta \leqslant \pi$. In practice, the angles have been grouped into five directions from forward to backward $(0, \pi / 4, \pi / 2,3 \pi / 4, \pi)$.

The correlations were calculated at each point of the cluster and averaged separately over each seventh of the total number of particles in the order of growth (for CA there is no natural order of growth and they were simply grouped according to the distance from the particle closest to the centre of mass).

The reason for calculating the correlations separately for each set of particles (they form concentrical rings around the origin) is to distinguish the region of initial growth and the surface region from the scaling region(Eq. (1)). This is shown in figure 2 for the axial correlation $c(r=6, \theta)$.
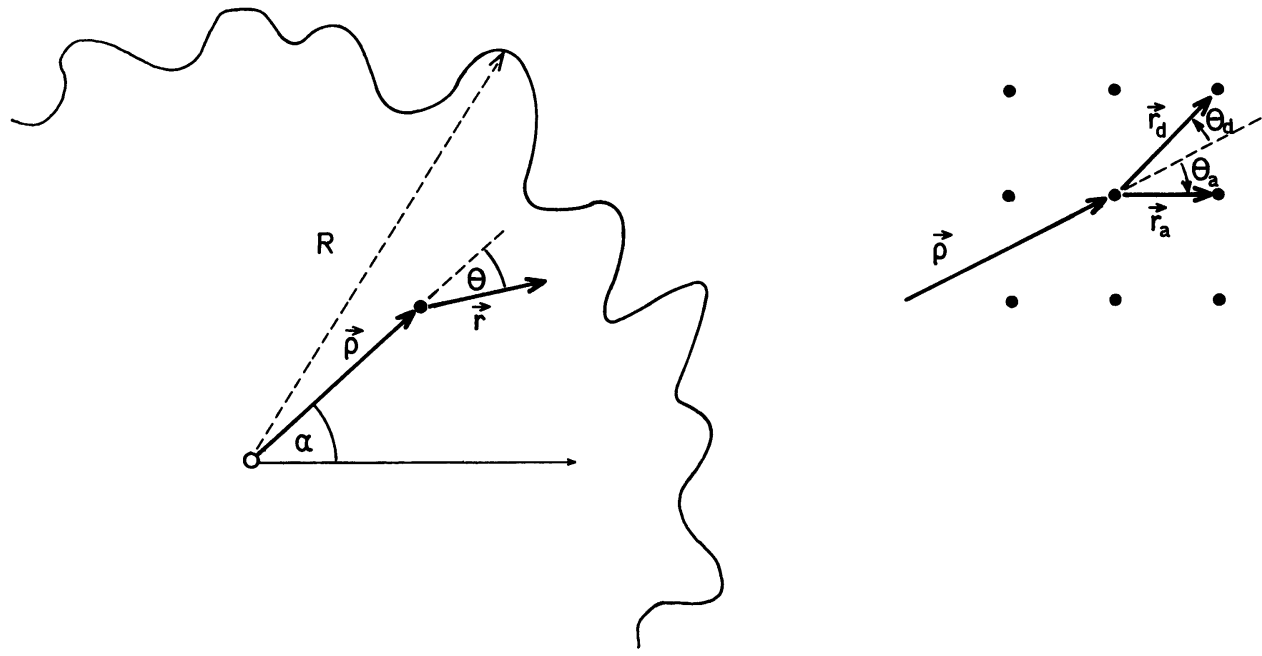

Fig. 1. - Definition of the angular correlations $c(\boldsymbol{\rho}, \mathbf{r})=c(r, \theta)$. From the origin (the seed particle for PA, the particle closest to the centre of mass for CA) $\rho$ denotes the coordinates of a particle of the cluster. The correlation functions $c(\boldsymbol{\rho}, \mathbf{r})=\langle n(\boldsymbol{\rho}+\mathbf{r}) n(\boldsymbol{\rho})\rangle=\langle n(\boldsymbol{\rho}+\mathbf{r})\rangle$ is the average density $n$ at $\boldsymbol{\rho}+\mathbf{r}$ and only depends on $r$ and $\theta$ (as shown on the left). On the lattice, $c(\boldsymbol{\rho}, \mathbf{r})$ also depends on the angle $\alpha$ between $\rho$ and one axis of the lattice, and $\mathbf{r}$ is restricted to points on the lattice. Axial $\left(\theta_{\mathrm{a}}\right)$ and diagonal $\left(\theta_{\mathrm{d}}\right)$ correlations are calculated here (as indicated on the right). 

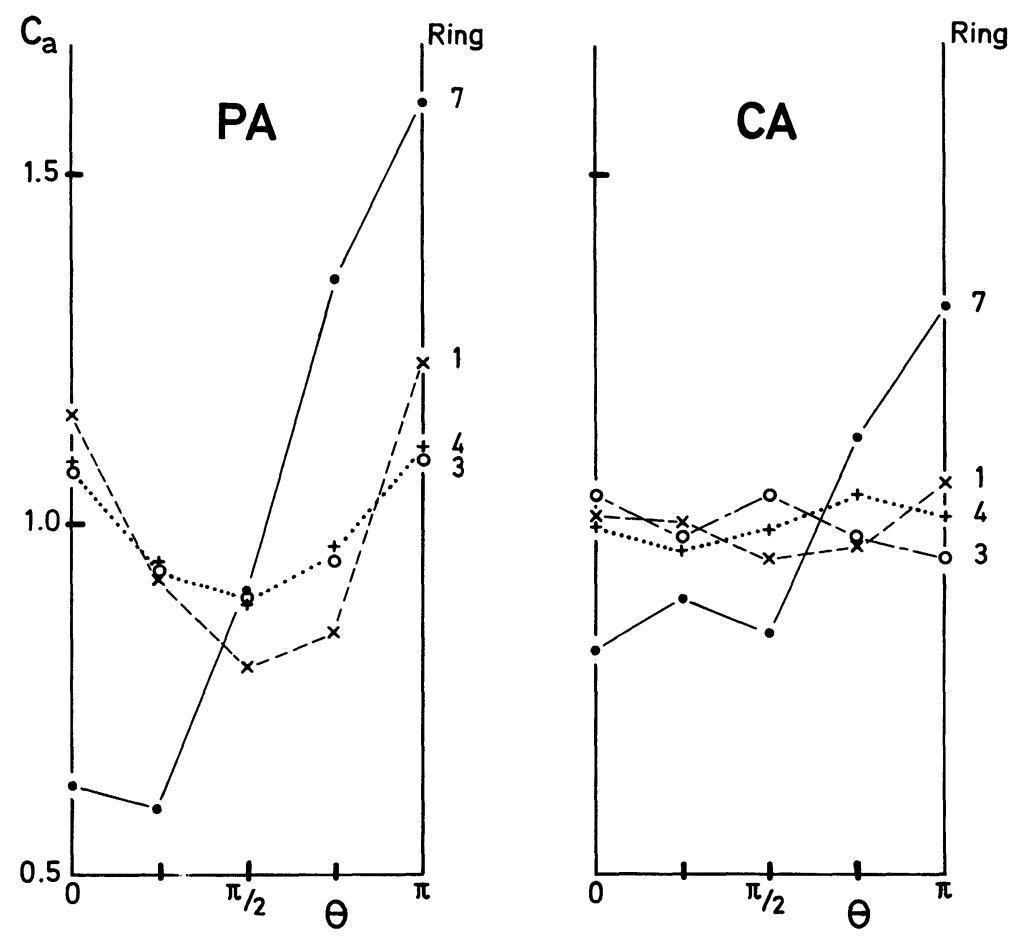

Fig. 2. - Axial correlations $c_{\mathrm{a}}(r=6, \theta)$ (normalized by the average of $c_{\mathrm{a}}$ over angles). They are calculated for all points on the cluster and then are averaged separately over concentrical rings with the same number of particles around the origin (ring 1-ring 7). Initial and surface effects clearly modify the angular dependence of $c_{\mathrm{a}}$. PA is shown on the left, CA on the right.

The distance $r$ is measured in lattice spacings. In ring three and four the angular correlations are the same (independent of $\rho$ ), which indicates that this is the scaling region. This conclusion is also supported from analysing clusters with half as many particles. Both initial effects due to the spherical geometry (ring 1, PA) and surface effects as the growth is incomplete (ring 7, PA and CA) modify the angular dependence of $c(\boldsymbol{\rho}, \mathbf{r})$. Similar results are obtained for other values of $r$ and for the diagonal correlation function.

In figure 3, the axial and diagonal correlations in the scaling region are shown as a function of $\theta$ with $r$ as a parameter. PA (left) and CA (right) show an entirely different behaviour. For PA, as $r$ increases, the transverse $(\theta=\pi / 2)$ correlations steadily decrease in comparison with the longitudinal correlations $(\theta=0, \pi)$. For CA, on the other hand, there is no such trend. For PA and for CA, comparing axial with diagonal correlations shows that the lattice has a tendency to diminish (enhance) the correlations for $\alpha=\pi / 4(\alpha=0)$. For PA, figure 3 suggests that the correlation scales with a different power parallel respectively perpendicular to the direction of growth :

$$
c(r, \theta=0, \pi) \sim r^{-A_{\|}}, \quad c(r, \theta=\pi / 2) \sim r^{-A_{\perp}} .
$$

Plotting the ratio $[c(r, \theta=0)+c(r, \theta=\pi)] /[2 c(r, \theta=\pi / 2)]$ directly determines the difference $\Delta A=A_{\perp}-A_{\|}$. In figure 4 , this ratio is shown. The exponent $\Delta A=0.16 \pm 0.05$ is rather small. The same analysis for CA shows no difference between longitudinal and transverse scaling. 

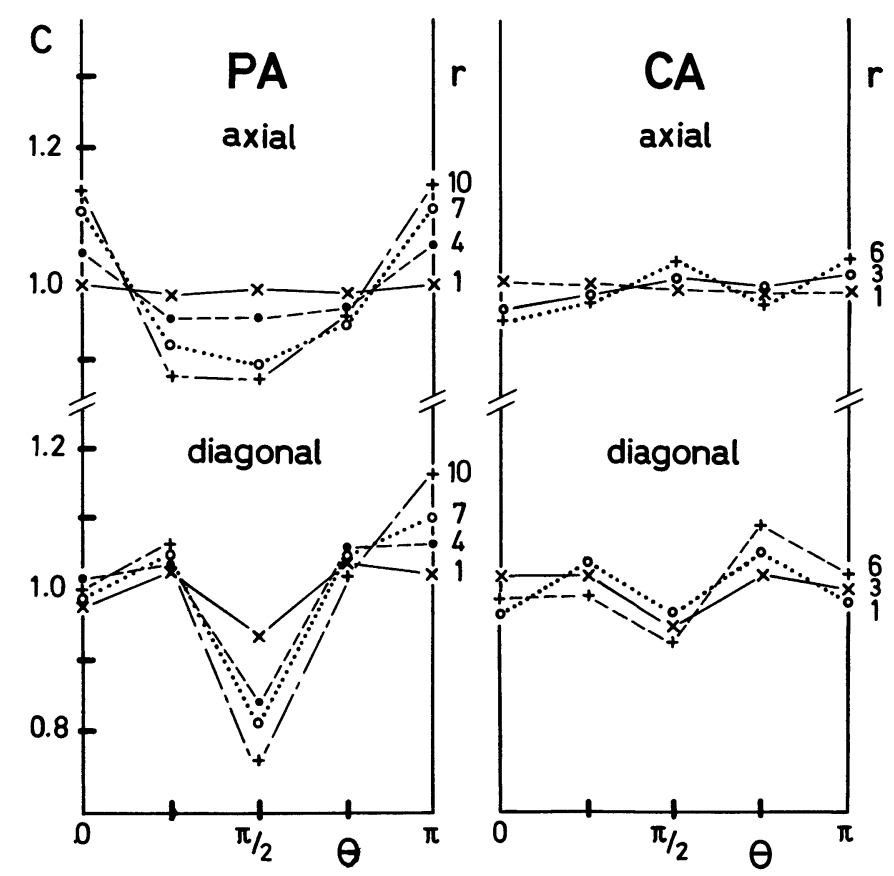

Fig. 3. - Axial and diagonal correlations $c(r, \theta)$ normalized for PA and CA as a function of the angle $\theta$ with $r$ as a parameter. The results are obtained deep inside the cluster, where equation (1) is valid (the correlations are averaged over the points of the fourth ring alone). The results for PA (left) differ markedly from CA (right). They suggest different scaling behaviour parallel and perpendicular to the growth direction, for PA only. Comparing the axial correlations (top) with the diagonal correlations (bottom) also show, that the lattice changes the amplitudes of the correlations, both for PA and for CA. The statistical error of the points is less than 0.04 . For the diagonal correlations $r$ is labelled in units of $\sqrt{2}$ times the lattice spacing.

The anisotropy found for the PA clusters has a number of consequences. First of all, it introduces correction to scaling effects which can be observed by plotting the axial and diagonal correlations, averaged over all angles. In figure $5, c(r)=\overline{c(r, \theta)^{\theta}}$ is shown as a function of $r$. For PA, the initial slope for $c_{\mathrm{a}}$ is much steeper than the one for $c_{\mathrm{d}}$. It suggests that the behaviour of $c_{\mathrm{a}}$ initially $(A=0.42)$ is determined by the transverse exponent before it crosses over to the asymptotic longitudinal behaviour. In contrast, $c_{\mathrm{d}}$ scales with an exponent $(A=0.28)$ close to the expected longitudinal exponent from the start. This shows that the lattice effects influence the two correlations differently, shifting the onset of the asymptotic scaling region. For CA, corrections to scaling are much weaker.

Usually, lattice effects are not expected to change the asymptotic scaling properties in critical phenomena [5]. Here however, while they don't change the exponents, they do change the critical amplitudes. This has been suggested recently for PA based on the visual appearance of very large clusters [6]. This conjecture is quantified by the results presented here, which indicate that the different shape of $c_{\mathrm{a}}(r, \theta)$ and $c_{\mathrm{d}}(r, \theta)$ persists when $r$ increases. More directly, the anisotropy of the clusters due to the lattice can be made quantitative by plotting the radius $R(N, \alpha)$ of the $N$ th aggregating particle as a function of the angle $\alpha$ at which it aggregates. In order to make $R(N, \alpha)$ independent of $N$, it is normalized by its angular average. Figure 6 shows clearly that the clusters are not spherical. The asymptotic behaviour is reached very slowly indicating a small correction to scaling exponent. In fact it is consistent with the idea, that the clusters reach 


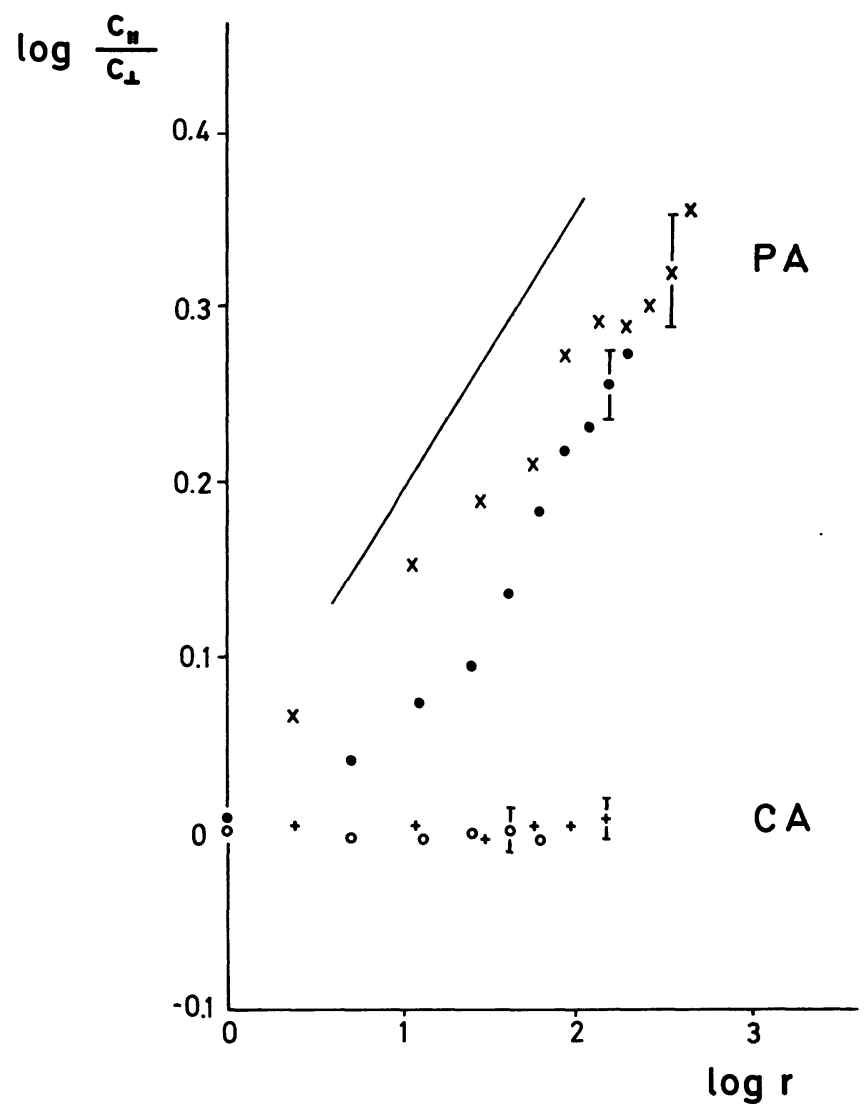

Fig. 4. - Log-Log plot of the ratio $c_{\|} / c_{\perp}=\frac{c(r, \theta=0)+c(r, \theta=\pi)}{2 c(r, \theta=\pi / 2)}$ versus $r$ for PA to determine the exponent $\Delta A=A_{\perp}-A_{\|}=0.16 \pm 0.05$. The straight line has slope 0.16 . The curves from axial and diagonal correlations gradually approach the same slope for large $r$. The same ratio for CA suggests $\Delta A=0$. The scaling region breaks down for $r \widetilde{>} 30(r \approx 20)$ for axial (diagonal) correlations, primarily as the edge of the cluster is reached (PA). The symbols stand for axial $(\bullet)$ and diagonal $(x)$ correlations for PA and for axial $(O)$ and diagonal $(+)$ correlations for CA.

their asymptotic shape with the same anisotropy exponent $\Delta A$ as observed above when comparing axial and diagonal correlations in figure 5 .

One important consequence of the difference between $A_{\|}$and $A_{\perp}$ is that a new length, $R_{\perp}$, is introduced in the PA process, which sets the scale perpendicular to the direction of growth, just as $R$ sets the scale radially. It can be obtained from the following simple argument : the mass in a ring of width $\Delta R$ at $R$ is $\sim \Delta R R^{1-A_{\|}}$but it is also $\Delta R\left(R / R_{\perp}\right) R^{1-A_{\perp}}$ where $R_{\perp}$ is the size of the scaling region in the transverse direction. This determines $R_{\perp} \sim R^{1-\Delta A}$ which means that the transverse scaling region gradually decreases with respect to $R$. With other words the angle $\Delta \alpha$ within which scaling holds perpendicular to the direction of growth goes to zero as $R^{-\Delta A}$ when $R \rightarrow \infty$.

The present study shows that by a more detailed analysis than the traditional fractal analysis for aggregation clusters, it is possible to determine quantitatively even slight anisotropies in scaling properties and in cluster shape on a lattice. Treating both cluster and particle aggre- 

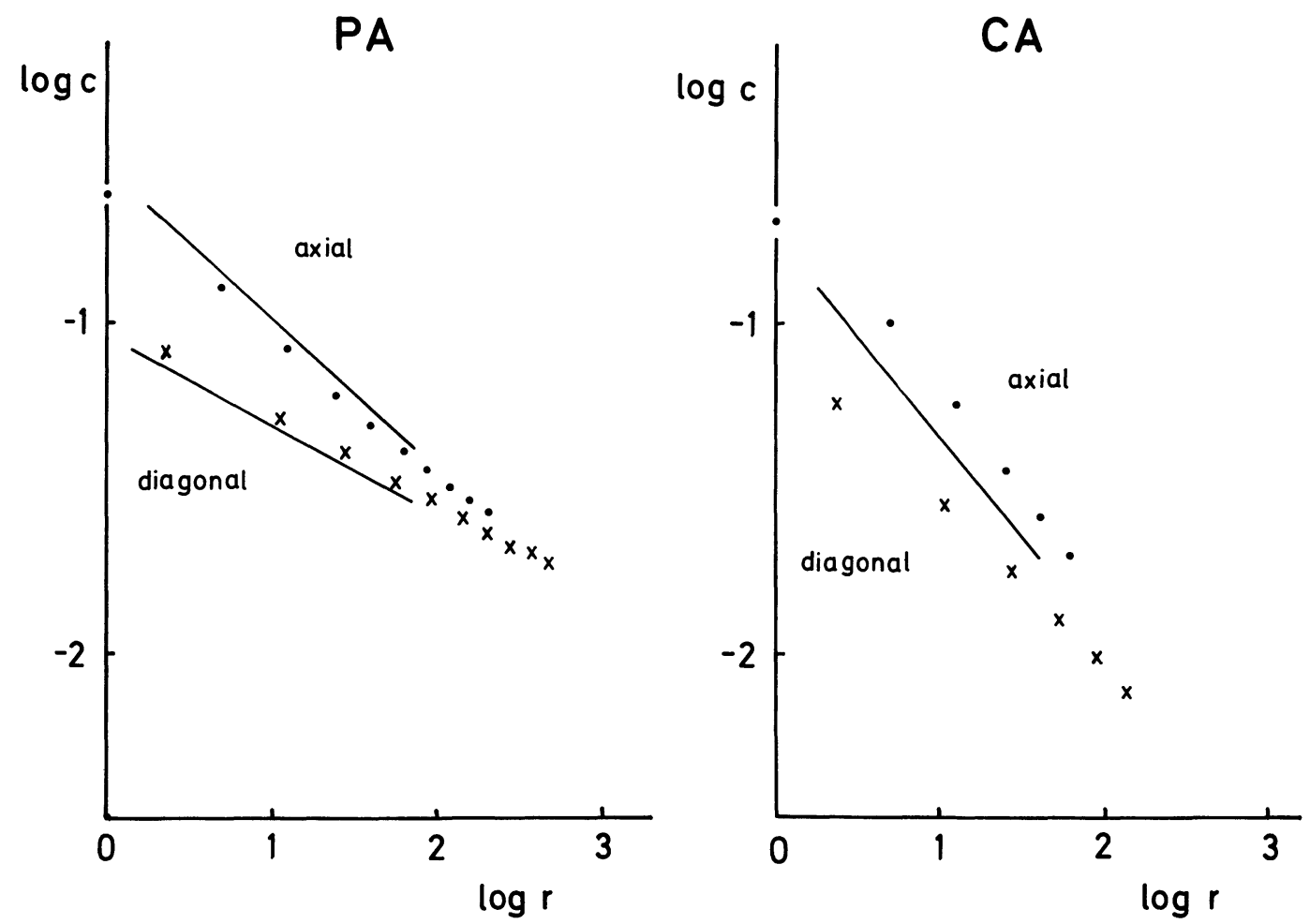

Fig. 5. - Log-Log plot of axial and diagonal correlations $c(r)$, averaged over the angles. For PA, axial and diagonal correlations have a different initial slope, reflecting the difference between the exponents $A_{\perp}$ and $A_{\|}$. For CA, the difference between axial and diagonal correlations is much smaller.

gation strengthens the case for the anisotropy for PA, as CA behaves distinctly different - even for modest cluster sizes.

Note added in proof. - The anisotropy of PA clusters has recently been investigated independently by $\mathrm{P}$. Meakin and T. Vicsek and by R. Voss, with conclusions similar to the ones obtained here.

\section{Acknowledgments.}

I have benefitted from stimulating discussions with R. Jullien and R. Botet. The computations have been supported by an Action Thématique Programmée (ATP) of the C.N.R.S. and by the Centre de Calcul Vectoriel pour la Recherche (CCVR), Palaiseau. 


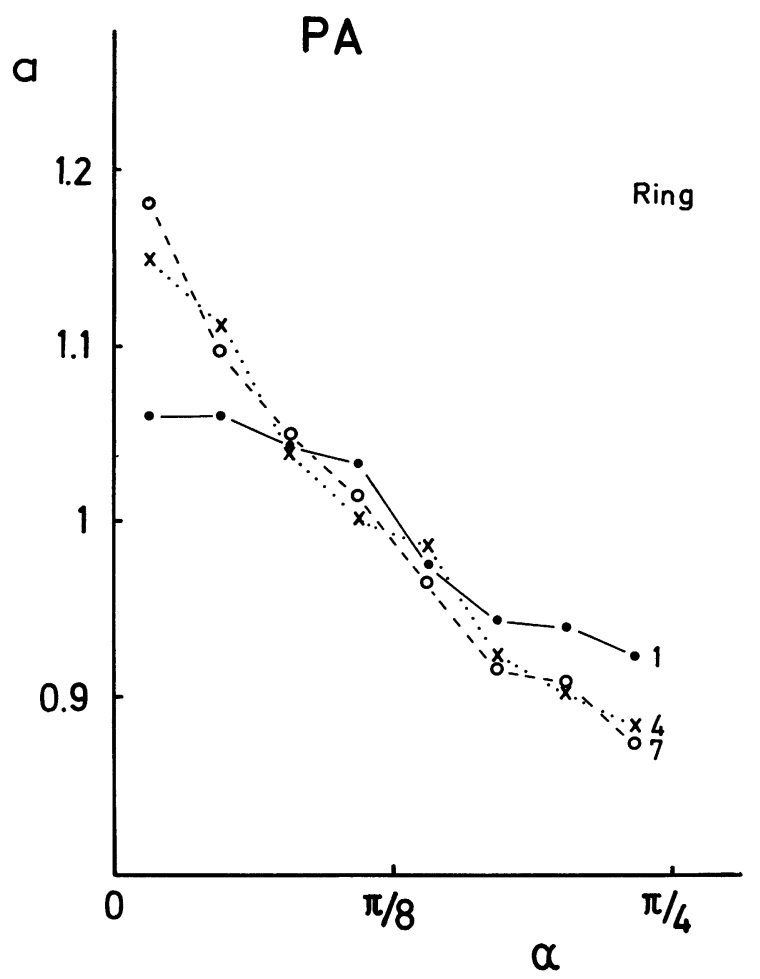

Fig. 6. $-R(N, \alpha)$ is the average radius of the $N$ th particle aggregating at an angle $\alpha$ on a square lattice. It is shown normalized, $a(\alpha)=R^{2}(N, \alpha) / R^{2}(N)$ where $R^{2}(N)$ is the angular average of $R^{2}(N, \alpha)$, and averaged separately for increasing rings, for PA, in the interval $0 \leqslant \alpha \leqslant \pi / 4$. It clearly shows the anisotropy of the shape of the clusters. The statistical errors are less than 0.03 .

\section{References}

[1] For a review see the abstracts of the workshop on kinetic models for cluster formation (september 17-28, 1984, CECAM Orsay); R. Jullien, M. Kolb, H. Herrmann and J. Vannimenus eds., J. Stat. Phys. (April 1985).

[2] Witten, T. A. and Sander, L. M., Phys. Rev. Lett. 47 (1981) 1400.

[3] Meakin, P., Phys. Rev. Lett. 51 (1983) 1119.

Kolb, M., Botet, R. and Jullien, R., Phys. Rev. Lett. 51 (1983) 1123.

[4] Botet, R., Jullien, R. and Kolb, M., J. Phys. A 17 (1984) L75.

[5] Wu, T. T. and McCoy, B. M., The two dimensional Ising model (Harvard) 1973.

[6] BraDy, R. and BALL, R., unpublished. They suggest that large PA clusters on a square lattice visually have the shape of a diamond. 